\title{
The Wind Momentum - Luminosity Relationship of Blue Supergiants
}

\author{
Rolf-Peter Kudritzki
}

Institut für Astronomie und Astrophysik der Universität München, Scheinerstr.1, D-81679 München, Germany

Max-Planck-Institut für Astrophysik, D-85740 Garching bei München, Germany

\begin{abstract}
The prediction of the Wind Momentum - Luminosity Relationship (WLR) based on the theory of radiation driven winds is verified by quantitative spectroscopy of winds of A-, B- and O-supergiants. The relationship depends on spectral type. New stellar wind calculations are presented reproducing the observed spectral type dependence. The impact of spectral variability on the WLR is investigated by an analysis of some hundred spectra of the luminous A0Ia-supergiant HD92207 obtained within the Heidelberg Spectral Variability Survey and found to be small.

Finally, the WLR is discussed as a tool for the determination of extragalactic distances. Recent results obtained for the Galaxy, M31 and M33 are presented. The potential of the method is discussed with the conclusion that it may allow independent distance moduli to be obtained with an accuracy of ten percent out to the Virgo and Fornax clusters of galaxies.
\end{abstract}

\section{The Wind Momentum - Luminosity Relationship}

The Wind Momentum - Luminosity Relationship (WLR) provides a new independent tool to derive extragalactic distances with an accuracy comparable to the Cepheid method. The basic concept is simple. Since the winds of the blue supergiants are a result of radiation pressure, we expect the mechanical momentum flow of a stellar wind $\dot{M} v_{\infty}$ to be a function of the photon momentum rate $L / c$ provided by the stellar photosphere and interior. Indeed, a straightforward analytical solution of the hydrodynamic equations of radiation driven winds yields (see Kuddritzki et al. 1989, Kudritzki et al. 1996a, Puls et al. 1996, Kudritzki 1998)

$$
\dot{\mathbf{M}} \mathbf{v}_{\infty} \propto \frac{1}{R_{*}^{0.5}} \mathbf{L}^{1 / \alpha}
$$

where $\alpha(\approx 2 / 3 \ldots 1 / 2)$ is well determined by atomic physics and represents the power law exponent of the distribution function of line strengths of the many thousands of lines driving the wind.

Thus, by measuring the rate of mass-loss and the terminal velocity directly from the spectrum we are - in principle - able to determine the luminosity 

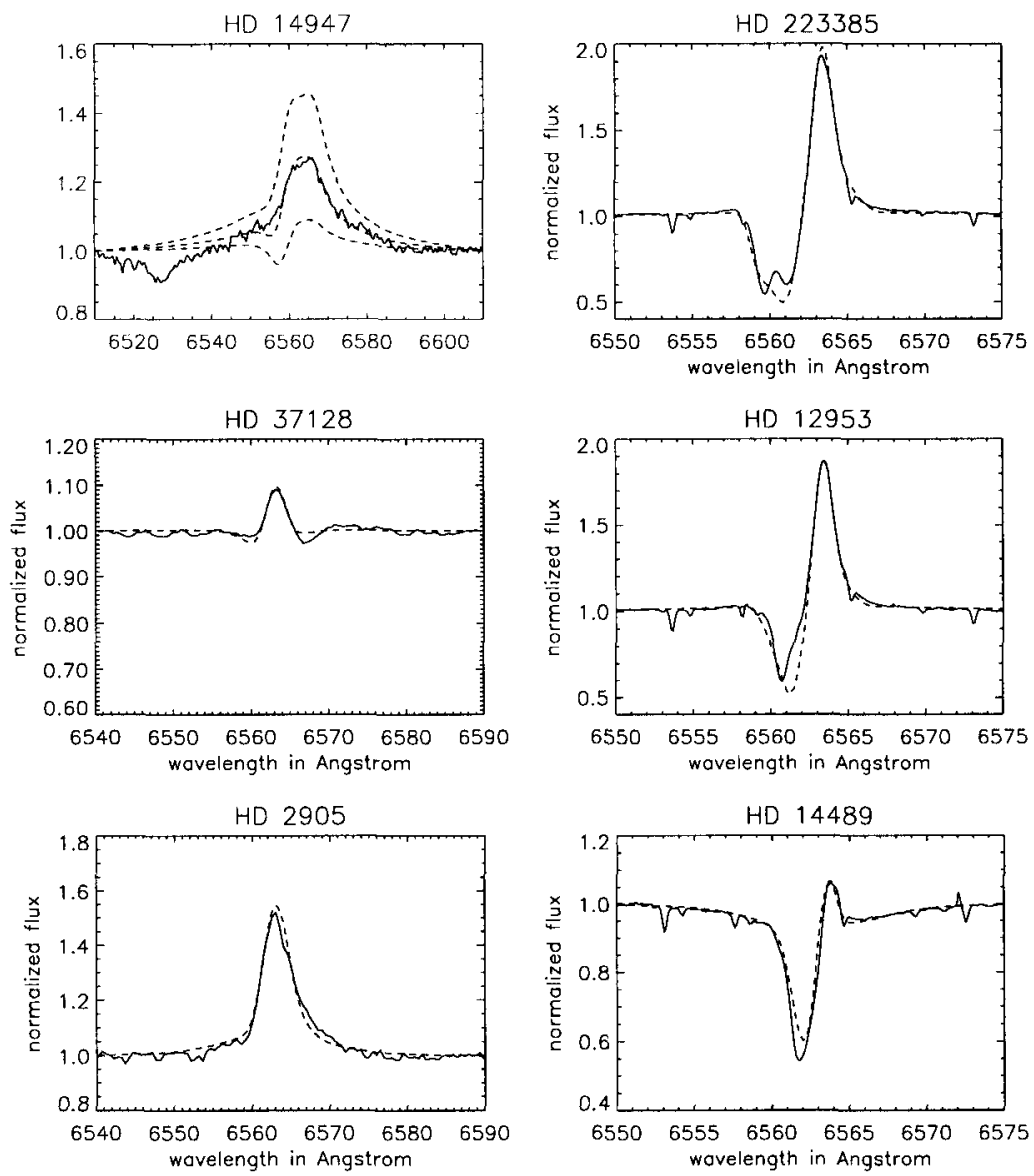

Fig. 1. $H_{\alpha}$ line profile fits of the galactic supergiants HD 14947 (O5 Ia), HD 37128 (B0 Ia), HD 2905 (B0.7 Ia), HD 223385 (A3 Ia), HD 12953 (A1 Ia), HD 14489 (A2 Ia). The fits of HD 14947 are taken from Puls et al., 1996 and the three different curves indicate adopted changes in the model calculations of 25 percent in $\dot{M}$. The fits of all other objects are from Kudritzki et al. et al., 1998, who used an improved version of the unified model atmosphere code developed by Santolaya-Ray, Puls and Herrero (1997) for the line profile fits.

of a blue supergiant. This is an exciting perspective, because it would give us a purely spectroscopic tool to determine stellar distances. Quantitative spectroscopy would yield $T_{\text {eff }}$, gravity, abundances, intrinsic colours, reddening, extinction, $\dot{M}$ and $v_{\infty}$. With the luminosity from the above relation one could then compare with the dereddened apparent magnitude to derive a distance.

It is, of course, challenging to compare the prediction of radiation driven wind theory with the observations to see whether an observed WLR does 

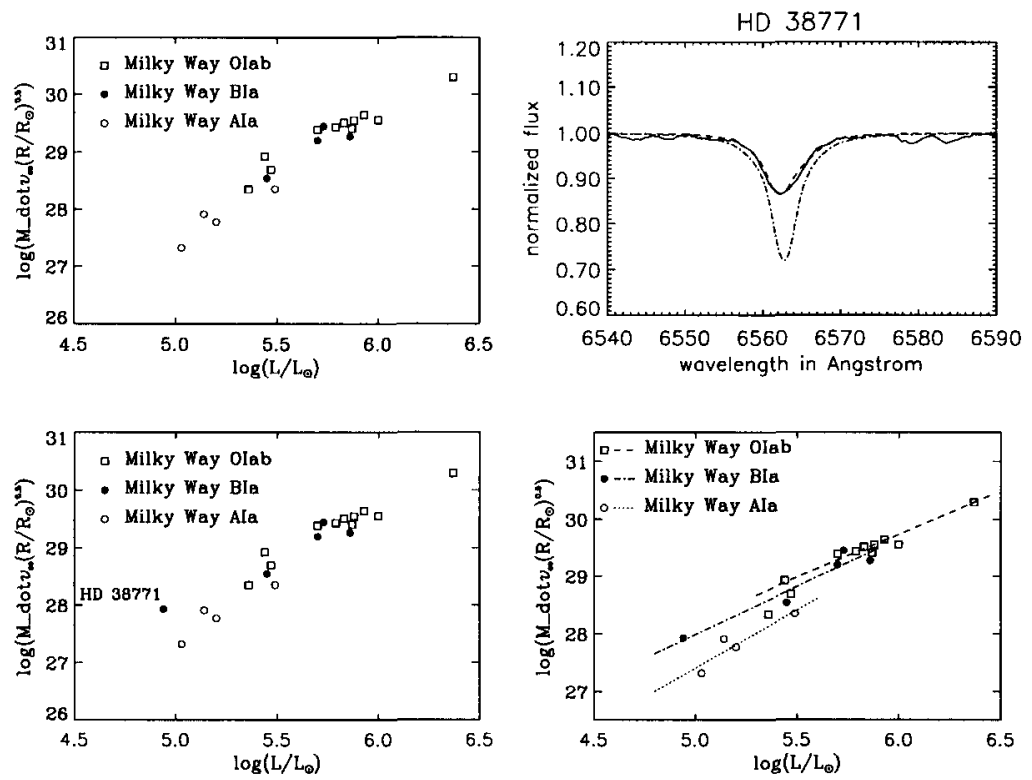

Fig. 2. Upper left: The observed WLR of the brightest galactic supergiants of spectral type O, early B and early A. Data from Puls et al., 1996 and Kudritzki et al. 1998. Wind momenta are given in cgs-units. Upper right: $\mathrm{H}_{\alpha}$ profile fit of the low luminosity supergiant HD 38771 ( $\kappa$ Ori, B0.5 Ia) with $\dot{M}=2.8$ and $1.010^{-7}$ $\mathrm{M}_{\odot} / \mathrm{yr}$ (dotted and dashed-dotted, respectively). Lower left: The observed WLR including HD 38771. Lower right: Three independent linear regressions fitted to the three spectral types assuming a temperature dependence of the WLR.

exist. This requires the determination of $\mathrm{v}_{\infty}$ and $\dot{M}$. While the measurement of the former is easy and straightforward by means of the blue edges of the $\mathrm{P}$-Cygni profiles of UV resonance lines (for O-, B-, and A-supergiants) or $\mathrm{H}_{\alpha}$ (for A-supergiants with winds of significant strength; for examples and references, see for instance Kudritzki 1998), the determination of the latter with sufficient precision used to be problematic. However, the development of NLTE Unified Model Atmospheres made it possible to use the strength of $\mathrm{H}_{\alpha}$ as a wind line to determine $\dot{M}$ very precisely (see again Kudritzki 1998 for an overview). In this way, Puls et al. 1996 obtained accurate mass-loss rates for a large sample of galactic $\mathrm{O}$-stars and provided a detailed discussion of the $\mathrm{H}_{\alpha}$ line formation process and the possible error sources. Kudritzki et al. $1998 \mathrm{~b}$ extended this work on the brightest galactic B- and A-supergiants with well determined distances. Fig. 1 indicates how accurately the observed $\mathrm{H}_{\alpha}$ stellar wind line profiles can be reproduced by the theoretical models yielding mass-loss rates with a precision better than 0.1 dex. Fig. 2 (upper left) combines the results from Puls et al. 1996 and Kudritzki et al. 1998b 
with regard to the observed wind momentum and reveals the clear existence of a WLR.

There is the indication of a slight curvature of the observed WLR or of the existence of three independent relations of slightly different height and slope for the three spectral types. The latter interpretation is supported by the investigation of the wind momentum of the B0.5 supergiant HD 38771 ( $\kappa$ Ori). This object is usually considered as a twin of HD $37128\left(\epsilon\right.$ Ori, $\mathrm{H}_{\alpha}$ profile displayed in Fig. 1), however, the recent astrometric results obtained with the Hipparcos satellite show that it is in the foreground of the Orion association with a de-reddened absolute magnitude of only $\mathrm{M}_{V}=-4.8^{m}$. This explains, why - contrary to HD $37128-\mathrm{H}_{\alpha}$ is in absorption and is fitted with a significantly smaller rate of mass-loss (see Fig. 2, upper right). The wind momentum of HD 38771 - a low luminosity B-supergiant - is still larger than the wind momentum of the A-supergiants of comparable luminosity (see Fig. 2, lower left). We take this as an indication of a temperature dependence of the WLR and adopt three independent linear regression for the three spectral types (Fig.2, lower right). We also note that the WLR of Central Stars of Planetary Nebulae, which are as hot or even hotter than O-star, follows the WLR of O-stars (see Kudritzki et al. 1997). This confirms our conclusion that the WLR is temperature dependent.

\section{New wind models for supergiants of spectral type $O$, $B$ and $A$}

To be able to investigate the systematic behaviour of line driven winds across the whole HRD of hot stars (where "hot" means that hydrogen is ionized and can be as cool as $\mathrm{T}_{\mathrm{eff}}=8000 \mathrm{~K}$ for A-supergiants) in all different stages of evolution (including massive stars as well as post-AGB) we have developed a new approach to calculate the wind dynamics. This approach is based on the improvements achieved during the last decade with regard to atomic physics and line lists (see Pauldrach et al. 1998). We use the line list of $2.510^{6}$ lines of 150 ionic species and apply analytical formulae (see Springmann 1997, Springmann and Puls 1998) for a fast approximation of NLTE occupation numbers to calculate the radiative line acceleration, which is then represented by a new parameterization using depth dependent force multiplier parameters (see Kudritzki et al. 1998a; note that now as an improvement real model atmosphere fluxes are used instead of Planck functions for the photospheric irradiation of the wind). Because of the depth dependent force multipliers a new formulation of the critical point equations is introduced (Kudritzki et al. 1998c). In this way, wind models can be calculated within a few seconds on a workstation for every hot star with specified effective temperature, mass, radius and abundances.

We have used this new approach to calculate wind models for typical parameters of $\mathrm{A}-, \mathrm{B}$ - and $\mathrm{O}$-supergiants. The results are compared with the 
observations in Fig. 3. Although much more work has yet to be invested in the future to compare theory and observation on a star by star basis, a first conclusion is that the theory is roughly able to reproduce the observed trends with regard to wind momentum and terminal velocity.
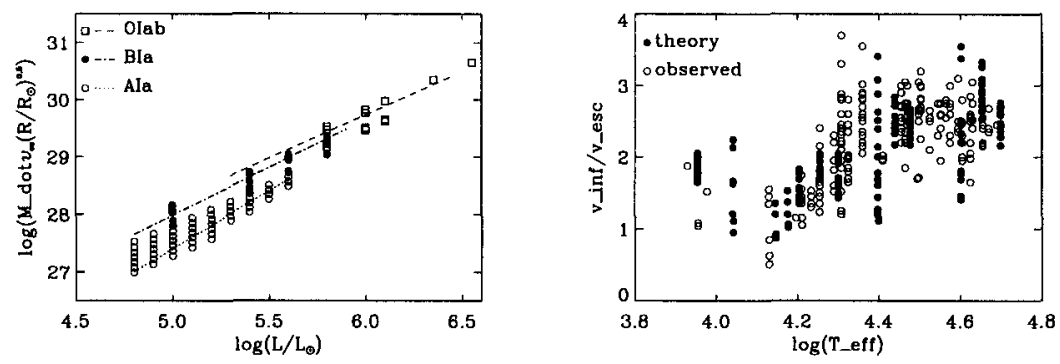

Fig. 3. New wind calculations compared with the observations. Left: Wind momenta as function of luminosity. The dotted, dashed-dotted and dashed curves correspond to the observed regression curves of Fig. 14 for A-, B- and O-supergiants, respectively. The (open and filled) circles and squares correspond to the models. Their scatter at a given luminosity is a result of the different masses or effective temperatures adopted. Right: Ratio of terminal velocity to photospheric escape velocity as function of effective temperature. Open circles represent observations taken from Prinja and Massa (1998). Filled circles correspond to the calculations and the scatter at a given temperature is a result of the different masses and luminosities which were adopted.

\section{Spectral variability - the case of the A0Ia-supergiant HD 92207}

The observed tight correlation of stellar wind momentum with luminosity seems to in variance with the variability of stellar wind lines observed in the spectra of many supergiants indicating stellar wind momenta to be a function of time. However, even strong changes in the observed line profiles of - for example $-\mathrm{H}_{\alpha}$ do not neccessarily transform into large changes of stellar wind momentum because of the extremely strong dependence of $\mathrm{H}_{\alpha}$ on mass-loss. Ruppersberg et al. 1998 (see also Ruppersberg 1998) have investigated this question by analyzing some hundred spectra of the the A0Ia-supergiant HD 92207 obtained within the Heidelberg Spectral Variability Survey by Kaufer et al. 1996 over a period of 150 days. For each spectrum effective temperature, gravity, radius, mass-loss rate and terminal velocity were carefully determined by line profile fits (see Fig. 4 for illustration). Mass-loss rates und terminal 

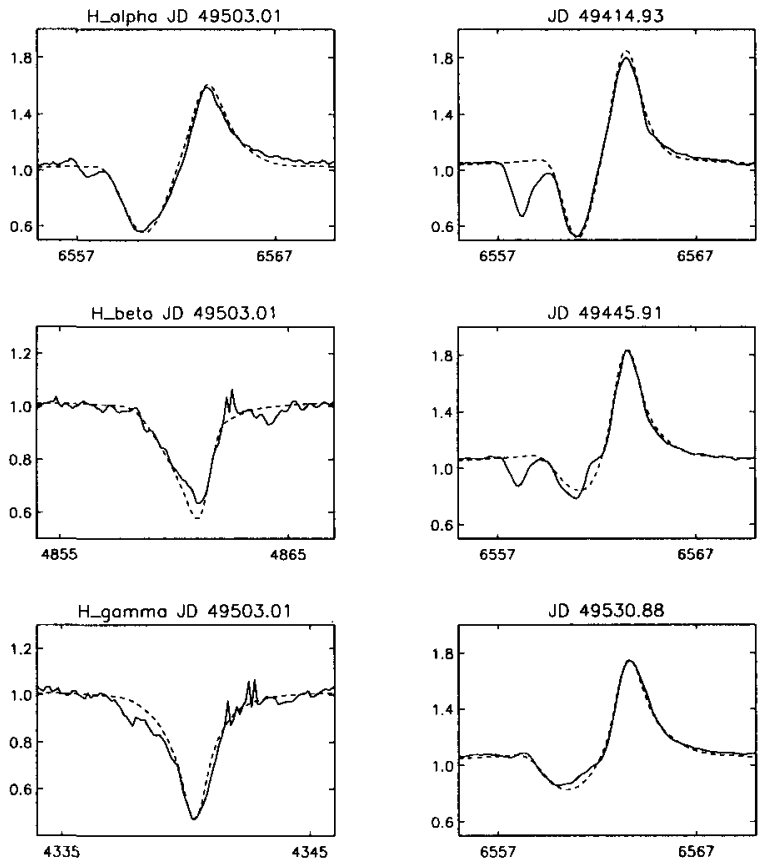

Fig. 4. Left: Simultaneous fit of the $\mathrm{H}_{\alpha}, \beta, \gamma$ profiles in one spectrum of HD 92207. Right: $\mathrm{H}_{\alpha}$ fits at three different times. (From Ruppersberg et al., 1999).

wind velocities obtained from this analysis did indeed show a significant variation as function of time. However, the variation in wind momentum turned out to be smaller than 0.2 dex (see Fig. 5) resulting only in a very small shift of this object in the wind momentum - luminosity plane typical for the general scatter within the WLR.

\section{The Wind Momentum - Luminosity Relationship and the determination of extragalactic distances}

It has long been a dream of stellar astronomers to use the spectra of the most luminous blue stars to determine the distances to other galaxies. Now, the WLR might provide the means to fullfill this dream.The basic technique is to derive the stellar parameters (temperature, gravity, metallicity) spectroscopically from optical absorption lines (see Kudritzki 1998 for references), and then to model the $\mathrm{H}_{\alpha}$ profiles $\left(\rightarrow \dot{M}\right.$ for $\mathrm{O}-, \mathrm{B}$ - and A-supergiants, $\mathrm{v}_{\infty}$ for A-supergiants) and the UV P-Cygni line profiles ( $\rightarrow \mathrm{v}_{\infty}$ for O-, B- and A-supergiants) to obtain the wind momentum. Application of the empirically calibrated WLR appropriate to the spectroscopically determined metallicity 

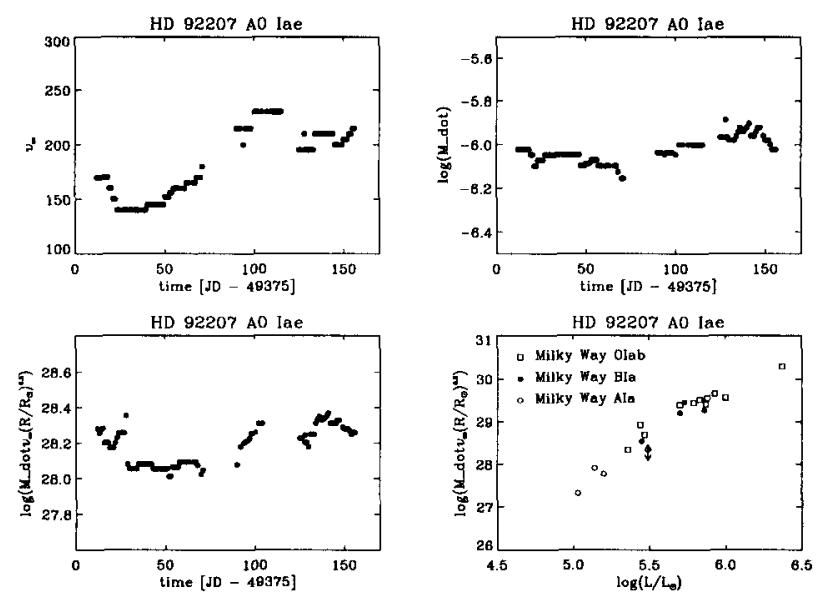

Fig. 5. Terminal velocity, mass-loss rate and stellar wind momentum of HD 92207 as function of time (upper left and right, lower left, respectively). The location of HD 92207 in the wind momentum - luminosity plane superimposed by the arrow indicating the maximum amount of momentum variability measured. (From Ruppersberg et al., 1999).

(for a discussion how to deal with different metallicities, see again Kudritzki 1998) then allows one to determine the intrinsic luminosity from which the distance follows once the stellar apparent magnitude and colours $(\rightarrow$ reddening and extinction in conjunction with the stellar parameters determined spectroscopically) are known. In practice, one will adopt a distance for all stars in a galaxy to locate them in the momentum/luminosity plane and will then iterate the distance until the distribution coincides with the calibrated sample.

\subsection{A first investigation in M31 and M33}

A crucial test of the WLR method is the investigation of wind momenta of blue supergiants in M31. In an extensive collaboration (see acknowledgements), we have started a systematic study using the WHT and Keck telescopes for optical spectroscopy and the HST for multicolour photometry and UV spectroscopy. While most of the Cycle 6 and 7 HST observations have still to be carried out, we are able to present the first analysis of two M31 A-supergiants (see Mc Carthy et al. 1997) based solely on Keck HIRES optical spectroscopy in Fig. 6 (see also Mc Carthy et al. 1995 for M33). The results are very encouraging. The $\mathrm{H}_{\alpha}$ line profiles allow a precise determination of wind momenta. Adopting a distance modulus of 24.25 mag (Rozanski \& Rowan-Robinson 1994) we find that both objects coincide very well with galactic WLR. Mc Carthy et al. 1997 estimate that with 10 to 20 objects 
it will be possible to obtain an independent M31 distance modules with an accuracy of $0.1 \mathrm{mag}$.
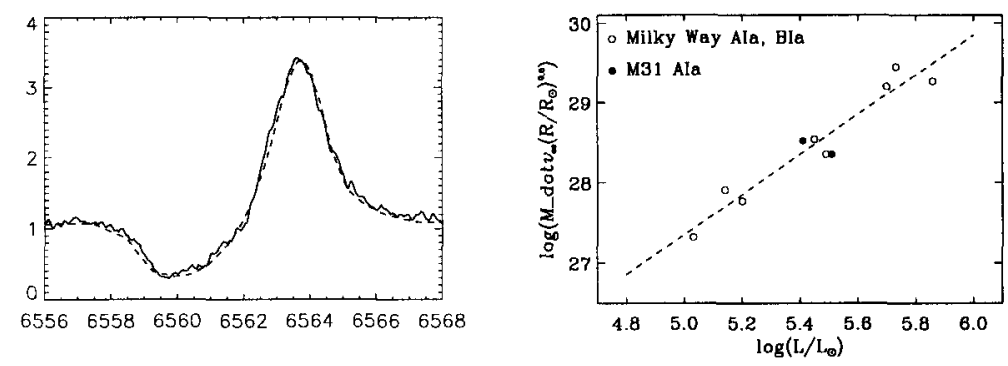

Fig. 6. Left: $\mathrm{H}_{\alpha}$ fit of the Keck HIRES spectrum of the M31 A2 Ia-O supergiant 41-3654 yielding a precise determination of $\dot{M}$ and $\mathrm{v}_{\infty}$. Right: Wind momenta of two A-supergiants in M31 (obtained with Keck HIRES) compared with galactic Aand B-supergiants of the solar neighbourhood. Results from McCarthy et al. 1997.

\subsection{The potential of the WLR-method}

The application of the WLR method does not neccessarily require spectra of high $\mathrm{S} / \mathrm{N}$ or high spectral resolution. As a very illustrative example we have used the $\mathrm{H}_{\alpha}$ and $\mathrm{H}_{\beta}$ equivalent widths of LMC A-supergiants published by Tully and Wolff 1984 to derive gravity and - most importantly - wind momenta (adopting $\mathrm{v}_{\infty}=150 \mathrm{~km} / \mathrm{s}$ for all objects). Fig. 7 shows the resulting correlation between wind momentum and absolute magnitude for those objects where a good simultaneous determination of gravity and mass-loss rate was possible (for details, see Knoerndel et al. 1998). Despite the limited quality of the data, the tightness of the relation obtained is striking.

If one wants to use this observed relationship to estimate the possible accuracy of distance determinations, it is important to realize that determining a mass-loss rate from an $\mathrm{H}_{\alpha}$ profile requires an assumption about the stellar radius and therefore the distance. Thus, both wind momentum and absolute magnitude depend on the radius adopted. However, as one can show analytically (Puls et al. 1996), the quantity

$$
Q=\dot{M} v_{\infty}\left(\frac{R_{*}}{R_{\odot}}\right)^{-1.5}
$$

is an invariant of the profile fitting, i.e. the strength of $\mathrm{H}_{\alpha}$ as a wind line depends exactly on this quantity. As a consequence, we also plot $Q$ as a function of $\mathrm{M}_{V}$ in Fig. 7 to quantify the distance independent scatter. The standard deviation in absolute magnitude from the mean relation $Q=f\left(M_{V}\right)$ 
is on the order of $0.3 \mathrm{mag}$. This confirms the estimate by Mc Carthy et al. 1997 that with ten to twenty objects per galaxy, distance moduli as accurate as $0.1 \mathrm{mag}$ should be achievable.
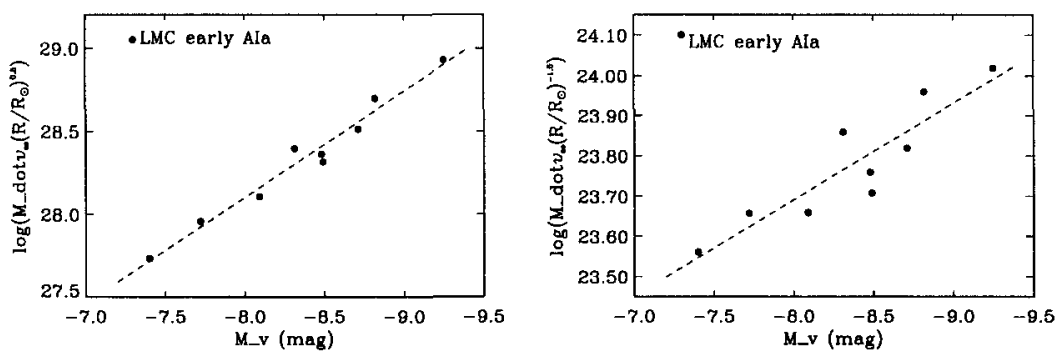

Fig. 7. Left: Wind momentum determined from the Balmer line equivalent widths of LMC AO Ia - supergiants published by Tully and Wolff (1984) as function of absolute magnitude (adopting a distance modulus of $18.5 \mathrm{mag}$ ). From Knoerndel et al., 1998. Right: Same as the left figure, but instead of wind momentum the invariant $\mathrm{Q}$ of the $\mathrm{H}_{\alpha}$ fitting (see text) is plotted.

The uncertainties in WLR distance moduli might, therefore, be comparable to those obtainable from Cepheids in galaxies. The advantage of the WLR-method, however, is that individual reddening (and therefore extinction) as well as metallicity can be derived directly from the spectrum of every object. Moreover, it is a new independent primary method for distance determination and can contribute to the investigation of systematic errors of extragalactic distances. However, the crucial question is to what distances will the method be applicable.

The best spectroscopic targets at large distances are A-supergiants. Since massive stars evolve at almost constant luminosity towards the red, A-supergiants are the optically brightest "normal" stellar objects because of the effects of Wien's law on the bolometric correction. In addition, for these objects we can determine the wind momentum solely by optical spectroscopy at $\mathrm{H}_{\alpha}$ (without need of the UV). This means that we can use ground-based telescopes of the $8 \mathrm{~m}$ class for spectroscopy rather than the tiny HST (which is then needed for accurate photometry only).

From Fig. 7 we see that the brightest A-supergiants have absolute magnitudes between -9 and -8 mag (objects like 41-3654 in M31 are another example). Such objects would be of apparent magnitude between 20 and 21 in galaxies $6 \mathrm{Mpc}$ away, certainly not a problem for medium $(2 \AA)$ resolution spectroscopy with $8 \mathrm{~m}$ class telescopes. Even in a galaxy like M100 at a distance of $16 \mathrm{Mpc}$ (Freedman et al. 1994b; Ferrarese et al. 1996) these objects would still be accessible at magnitudes around 22.5 and would yield wind mo- 
mentum distances if medium resolution spectroscopy on a $8 \mathrm{~m}$ class telescope were combined with HST photometry. Indeed, the HST colour magnitude diagram published by Freedman et al. 1994a may show the presence of such objects in M100.

One might ask, of course, whether a medium resolution of $2 \AA$ would still allow a sufficiently accurate determination of effective temperature, surface gravity, abundances, and wind momentum. We note that the rotational velocities of A-supergiants are typically on the order of $40 \mathrm{~km} \mathrm{~s}^{-1}$, a broadening which is matched by a spectral resolution of roughly $1.4 \AA$ at $\mathrm{H} \alpha$. Therefore we expect that the medium resolution situation will not be dramatically worse, provided sufficient S/N can be achieved and accurate sky-, galaxy-, and $\mathrm{H}$ II region-background subtraction can be performed at very faint magnitudes. Experiments with observed spectra degraded with regard to $\mathrm{S} / \mathrm{N}$, resolution and sky emission indicate that such observations are challenging but feasible.

In summary, the WLR results obtained so far in the Local Group galaxies are very encouraging. We are optimistic that after further tests and calibration steps, we will have a new distance determination method capable of reaching out as far as to the Virgo and Fornax clusters of galaxies.

\section{Acknowledgements}

It is a pleasure to thank Artemio Herrero and Ilu Monteverde from the IAC, Jim McCarthy (Caltech), Kim Venn (Minnesota), Stephen Smartt (La Palma) and the Munich crowd Rudi Gabler, Stefan Haser, Oliver Knoerndel, Margie and Danny Lennon (now at La Palma), Paco Najarro (now Madrid), Adi Pauldrach, Joachim Puls, Uwe Springmann and Gudrun Taresch for their contributions.

\section{References}

Ferrarese, L. et al. 1996, ApJ 464, 568

Freedman, W. et al. 1994a, ApJ 435, L31

Freedman, W. et al. 1994b, Nature 371, 757

Kaufer, A., Stahl, O., Wolf, B., Gäng, Th., Gummersbach, C.A., Kovacs, J., Mandel, H., Szeifert, Th. 1996, A\&A 305, 887

Knoerndel, O., Kudritzki, R.P., Puls, J., Lennon, D.J. 1998, A\&A, in preparation Kudritzki, R.P., Pauldrach, A.W.A., Puls,J., \& Abbott, D.C. 1989, A\&A 219, 205 Kudritzki, R.P., Lennon, D.J., Haser, S.M., Puls, J., Pauldrach, A., Venn, K., \& Voels, S.A. 1996a, in "Science with the Hubble Space Telescope II", eds. P. Benvenuti et al., 285-296

Kudritzki, R.P., Mendez, R.H., McCarthy, J.K., Puls, J. 1997, Proc. IAU Symp. 180, "Planetary Nebulae", eds. H.J. Habing and H.J.G.L. Lamers, page $64-74$, invited paper, (Kluwer Academic Publishers) 
Kudritzki, R.P., Springmann, U., Puls, J., Pauldrach, A.W.A., Lennon, M. 1998a, ASP Conf. Series Vol. 131, p. 299 - 308

Kudritzki, R.P., Puls, J., Lennon, D.J., Venn, K.A., McCarthy, J.K., \& Herrero, A. 1998b, A\&A, in preparation.

Kudritzki, R.P., Springmann, U., Puls, J., Pauldrach, A.W.A., Lennon, M. 1998c, $A \& A$, in preparation.

Kudritzki, R.P. 1998, "Quantitative Spectroscopy of the Brightest Blue Supergiant Stars in Galaxies" in Proc. of "Stellar Astrophysics for the Local Group", VIII Canary Island Winterschool for Astrophysics, eds. A. Aparicio et al., Cambridge University Press, p. 149-262, invited lectures

McCarthy, J.K., Lennon, D.J., Venn, K.A., Kudritzki, R.P., Puls, J., \& Najarro, F. 1995, ApJ 455, L35

McCarthy, J.K., Kudritzki, R.P., Lennon, D.J., Venn, K.A., Puls, J. 1997, ApJ 482, 757

Monteverde, I., Herrero, A., Lennon, D.J., \& Kudritzki, R.P. 1997, ApJ Letters 474, L107

Pauldrach, A.W.A., Lennon, M., Hoffmann, T.L., Sellmaier,F., Kudritzki, R.P., Puls, J. 1998, ASP Conference Series Vol. 131, page $258-277$

Prinja, R.K., Massa, D.L. 1998, ASP Conf. Series, Vol. 131, p. 218 - 227

Puls, J., Kudritzki, R.P., Herrero, A., Pauldrach, A., Haser, S.M., Lennon, D.J., Gabler, R., Voels, S.A., Vilchez, J.M., Wachter, S., \& Feldmeier, A. 1996, A\&A 305,171

Rozanski, R., \& Rowan-Robinson, M. 1994, MNRAS 271, 530

Ruppersberg, R., Kudritzki, R.P., Puls, J., Kaufer, A. 1998, in preparation for A\&A Ruppersberg, R. 1998, Diplomarbeit, University of Munich

Santolaya-Rey, E., Puls, J., \& Herrero, A. 1997, A\&A 323, 488

Springmann, U. 1997, doctoral thesis, University of Munich

Springmann, U., Puls, J. 1998, ASP Conf. Series, Vol. 131, p. 286 - 298

Tully, R.B., Wolff, S.C. 1984, ApJ 281, 67

\section{Discussion}

S. Owocki: One remarkable implication of the observed wind-momentumluminosity relation is that the line parameters are nearly universal for $O$, B, and A stars. But if you were to add WR stars to your observational comparison, they would not fit by a factor of ten. This suggests that there is something about WR winds that makes line opacity a factor of ten more efficient in driving mass. All this is quite independent of a single scattering momentum limit, but represents a kind of "opacity problem". 